\title{
The higher covariant derivative regularization as a tool for revealing the structure of quantum corrections in supersymmetric gauge theories.
}

\author{
K.V.Stepanyantz \\ Moscow State University, \\ Faculty of Physics, Department of Theoretical Physics, \\ 119991, Moscow, Russia
}

November 14, 2019

\begin{abstract}
We discuss why the Slavnov higher covariant derivative regularization appeared to be an excellent instrument for investigating quantum corrections in supersymmetric gauge theories. For example, it allowed to demonstrate that the $\beta$-function in these theories is given by integrals of double total derivatives and to construct the NSVZ renormalization prescription in all loops. It was also used for deriving the non-renormalization theorem for the triple gauge-ghost vertices. With the help of this theorem the exact NSVZ $\beta$-function was rewritten in a new form, which revealed its perturbative origin. Moreover, in the case of using the higher covariant derivative regularization it is possible to construct a method for obtaining the $\beta$-function of $\mathcal{N}=1$ supersymmetric gauge theories, which simplifies the calculations in a great extent. This method is illustrated by an explicit two-loop calculation made in the general $\xi$-gauge.
\end{abstract}

\section{Introduction}

Investigation of quantum corrections in various field theory models plays an important role for understanding nature. For instance, the comparison of the experimental values for the electron and muon anomalous magnetic moments with the theoretical predictions unambiguously indicates that nature should be described by quantum field theory [1]. The renormalization group behaviour of the running coupling constants in the Standard model and its extensions can be interpreted as an indirect evidence of supersymmetry and Grand Unification [2]. Certainly, there are also a large number of other examples. However, it is well known that usually quantum corrections are divergent in the ultraviolet region, so that for calculating them one should use a regularization. Although the most popular method for making perturbative calculations is dimensional regularization [3, 4, 5, 6, for supersymmetric theories it is very inconvenient because of the manifest breaking of supersymmetry [7]. Its special modification, called dimensional reduction [8], appears to be mathematically inconsistent [9] and can also break supersymmetry in very higher orders [10, 11]. However, for regularizing supersymmetric theories one can use generalizations of the higher covariant derivative regularization proposed by A.A.Slavov in Refs. [12, 13]. An evident advantage of this regularization is that it is formulated in integer space-time dimensions. Moreover, in the supersymmetric case it can be consistently formulated in terms of $\mathcal{N}=1$ superfields [14, 15]. It is also possible to construct $\mathcal{N}=2$ supersymmetric higher derivative regulators [16], but for theories with extended supersymmetry the version formulated 
in $\mathcal{N}=2$ harmonic superspace [17, 18] is the most preferable. It has been constructed in Ref. [19] and allows to calculate quantum corrections in a manifestly $\mathcal{N}=2$ supersymmetric way in all orders. Using this regularization one can derive the non-renormalization theorems for theories with extended supersymmetry [20, 21, 22, 23] in a simple and elegant way [19, 24].

The main idea of the higher covariant derivative regularization is to add a term with a large degree of the covariant derivatives to the classical action. This allows to remove all divergences beyond the one-loop approximation [25]. For regularizing the remaining one-loop divergences one has to insert into the generating functional the Pauli-Villars determinants [26]. The presence of higher derivatives in the action essentially complicates explicit calculations of quantum corrections. For a long time this was a main obstacle for using this regularization. However, the calculations made in supersymmetric theories during the last decades demonstrated that the higher derivative regularization reveals the structure of quantum corrections in these theories and allows to solve the long-standing problems of deriving the NSVZ equation and constructing the NSVZ scheme. In this paper we will briefly review these issues and illustrate the results by an explicit two-loop calculation.

\section{The supersymmetric version of the higher covariant derivative regularization}

It is convenient to formulate $\mathcal{N}=1$ supersymmetric theories in superspace, because in this case supersymmetry turns out to be a manifest symmetry. In this case a general massless renormalizable $\mathcal{N}=1$ supersymmetric gauge theory with a simple gauge group $G$ is described by the action

$$
\begin{aligned}
S_{\text {classical }}=\frac{1}{2 e^{2}} \operatorname{Retr} \int d^{4} x d^{2} \theta W^{a} W_{a} & +\frac{1}{4} \int d^{4} x d^{4} \theta \phi^{* i}\left(e^{2 V}\right){ }_{i}{ }^{j} \phi_{j} \\
& +\left(\frac{1}{6} \lambda^{i j k} \int d^{4} x d^{2} \theta \phi_{i} \phi_{j} \phi_{k}+\text { c.c. }\right),
\end{aligned}
$$

where $V$ is the gauge superfield, and $\phi_{i}$ are the chiral matter superfields. The supersymmetric gauge superfield strength $W_{a}=\bar{D}^{2}\left(e^{-2 V} D_{a} e^{2 V}\right) / 8$ is a chiral superfield which transforms as a right spinor under the Lorentz group.

To construct the corresponding quantum theory, it is convenient to use the background field method [27, 28, 29] in the supersymmetric version [20, 30. Moreover, it is necessary to take into account the necessity of the non-linear renormalization of the quantum gauge superfield, see the general consideration in Refs. [31, 32, 33, and explicit calculations of Refs. [34, 35, 36]. This can be done by making the substitution

$$
e^{2 V} \rightarrow e^{2 \mathcal{F}(V)} e^{2 \boldsymbol{V}}
$$

where the function $\mathcal{F}(V)$ contains an infinite set of parameters needed for making the nonlinear renormalization, and $\boldsymbol{V}$ is the background gauge superfield. Note that in this case the quantum gauge superfield satisfies the constrain $V^{+}=e^{-2 \boldsymbol{V}} V e^{2 \boldsymbol{V}}$.

To introduce the higher covariant derivative regularization we first modify the action in such a way that the regularized action contains higher degrees of the supersymmetric covariant derivatives,

$$
S_{\text {reg }}=\frac{1}{2 e_{0}^{2}} \operatorname{Retr} \int d^{4} x d^{2} \theta W^{a}\left(e^{-2 \boldsymbol{V}} e^{-2 \mathcal{F}(V)}\right)_{A d j} R\left(-\frac{\bar{\nabla}^{2} \nabla^{2}}{16 \Lambda^{2}}\right)_{A d j}\left(e^{2 \mathcal{F}(V)} e^{2 \boldsymbol{V}}\right)_{A d j} W_{a}
$$




$$
+\frac{1}{4} \int d^{4} x d^{4} \theta \phi^{* i}\left(F\left(-\frac{\bar{\nabla}^{2} \nabla^{2}}{16 \Lambda^{2}}\right) e^{2 \mathcal{F}(V)} e^{2 \boldsymbol{V}}\right)_{i}^{j} \phi_{j}+\left(\frac{1}{6} \lambda_{0}^{i j k} \int d^{4} x d^{2} \theta \phi_{i} \phi_{j} \phi_{k}+\text { c.c. }\right),
$$

where $e_{0}$ and $\lambda_{0}^{i j k}$ are the bare gauge and Yukawa coupling constants, respectively. In the notation adopted in this paper the supersymmetric covariant derivatives are defined as

$$
\nabla_{a}=\nabla_{a}=D_{a} ; \quad \bar{\nabla}_{\dot{a}}=e^{2 \mathcal{F}(V)} e^{2 \boldsymbol{V}} \bar{D}_{\dot{a}} e^{-2 \boldsymbol{V}} e^{-2 \mathcal{F}(V)} ; \quad \overline{\boldsymbol{\nabla}}_{\dot{a}}=e^{2 \boldsymbol{V}} \bar{D}_{\dot{a}} e^{-2 \boldsymbol{V}} .
$$

They are present inside the regulator functions $R(x)$ and $F(x)$ which should rapidly grow at infinity and satisfy the condition $R(0)=F(0)=1$. The simplest choice is $R(x)=1+x^{m}$, $F(x)=1+x^{n}$, where $m$ and $n$ are positive integers. In Eq. (3) the gauge superfield strength is

$$
W_{a} \equiv \frac{1}{8} \bar{D}^{2}\left(e^{-2 \boldsymbol{V}} e^{-2 \mathcal{F}(V)} D_{a}\left(e^{2 \mathcal{F}(V)} e^{2 \boldsymbol{V}}\right)\right) .
$$

As a gauge fixing action we use the term

$$
S_{\mathrm{gf}}=-\frac{1}{16 \xi_{0} e_{0}^{2}} \operatorname{tr} \int d^{4} x d^{4} \theta \nabla^{2} V K\left(-\frac{\bar{\nabla}^{2} \nabla^{2}}{16 \Lambda^{2}}\right)_{A d j} \bar{\nabla}^{2} V
$$

where $K(0)=1$ and $K(x) \rightarrow \infty$ at $x \rightarrow \infty$. The corresponding Faddeev-Popov and NielsenKallosh actions are written as

$$
\begin{aligned}
& S_{\mathrm{FP}}=\frac{1}{2} \int d^{4} x d^{4} \theta\left.\frac{\partial \mathcal{F}^{-1}(\widetilde{V})^{A}}{\partial \widetilde{V}^{B}}\right|_{\widetilde{V}=\mathcal{F}(V)}\left(\left(e^{2 \boldsymbol{V}}\right)_{A d j} \bar{c}+\bar{c}^{+}\right)^{A} \\
& \times\left\{\left(\frac{\mathcal{F}(V)}{1-e^{2 \mathcal{F}(V)}}\right)_{A d j} c^{+}+\left(\frac{\mathcal{F}(V)}{1-e^{-2 \mathcal{F}(V)}}\right)_{A d j}\left(\left(e^{2 \boldsymbol{V}}\right)_{A d j} c\right)\right\}^{B} ; \\
& S_{\mathrm{NK}}=\frac{1}{2 e_{0}^{2}} \operatorname{tr} \int d^{4} x d^{4} \theta b^{+}\left(K\left(-\frac{\bar{\nabla}^{2} \nabla^{2}}{16 \Lambda^{2}}\right) e^{2 \boldsymbol{V}}\right)_{A d j} b,
\end{aligned}
$$

respectively. Here $c=e_{0} c^{A} t^{A}$ and $\bar{c}$ are the chiral Faddeev-Popov ghost superfields, and the chiral superfield $b=e_{0} b^{A} t^{A}$ stands for the Nielsen-Kallosh ghosts. Note that after the gauge fixing procedure the total action $S_{\text {total }}=S_{\text {reg }}+S_{\mathrm{gf}}+S_{\mathrm{FP}}+S_{\mathrm{NK}}$ remains invariant under the background gauge transformations and the BRST transformations [37, 38] in the superfield version [31.

The one-loop divergences are regularized by inserting into the generating functional the relevant Pauli-Villars determinants [26]. In the supersymmetric case, following Ref. [39], the generating functional for the regularized theory can be constructed as

$$
Z=\int D \mu \operatorname{Det}\left(P V, M_{\varphi}\right)^{-1} \operatorname{Det}(P V, M)^{c} \exp \left\{i\left(S_{\text {reg }}+S_{\text {gf }}+S_{\mathrm{FP}}+S_{\mathrm{NK}}+S_{\text {sources }}\right)\right\},
$$

where $D \mu$ is the measure of the functional integration and

$$
\operatorname{Det}\left(P V, M_{\varphi}\right)^{-1} \equiv \int D \varphi_{1} D \varphi_{2} D \varphi_{3} \exp \left(i S_{\varphi}\right) ; \quad \operatorname{Det}(P V, M)^{-1} \equiv \int D \Phi \exp \left(i S_{\Phi}\right) .
$$

In the first determinant $\varphi_{a}$ with $a=1,2,3$ are the commuting chiral superfields in the adjoint representation of the gauge group, which cancel one-loop divergences introduced by gauge and ghost loops. The second determinant contains commuting chiral superfields $\Phi_{i}$ in the representation $R_{\mathrm{PV}}$ which admits the gauge invariant mass term with $M^{j k} M_{k i}^{*}=M^{2} \delta_{i}^{j}$. (For example, 
it is possible to choose the adjoint representation.) These superfields cancel the one-loop divergences coming from the loop of the matter superfields $\phi_{i}$ for $c=T(R) / T\left(R_{\mathrm{PV}}\right)$, where $T(R)$ is defined by the equation $\operatorname{tr}\left(T^{A} T^{B}\right)=T(R) \delta^{A B}$. To regularize the one-loop supergraphs, the actions for the Pauli-Villars superfields should be written as

$$
\begin{aligned}
& S_{\varphi}=\frac{1}{4} \int d^{4} x d^{4} \theta\left\{\varphi_{1}^{* A}\left[\left(R\left(-\frac{\bar{\nabla}^{2} \nabla^{2}}{16 \Lambda^{2}}\right) e^{2 \mathcal{F}(V)} e^{2 \boldsymbol{V}}\right)_{A d j} \varphi_{1}\right]_{A}+\varphi_{2}^{* A}\left[\left(e^{2 \mathcal{F}(V)} e^{2 \boldsymbol{V}}\right)_{A d j} \varphi_{2}\right]_{A}\right. \\
& \left.+\varphi_{3}^{* A}\left[\left(e^{2 \mathcal{F}(V)} e^{2 \boldsymbol{V}}\right)_{A d j} \varphi_{3}\right]_{A}\right\}+\left(\frac{1}{4} M_{\varphi} \int d^{4} x d^{2} \theta\left(\left(\varphi_{1}^{A}\right)^{2}+\left(\varphi_{2}^{A}\right)^{2}+\left(\varphi_{3}^{A}\right)^{2}\right)+\text { c.c }\right) ; \\
& S_{\Phi}=\frac{1}{4} \int d^{4} x d^{4} \theta \Phi^{* i}\left(F\left(-\frac{\bar{\nabla}^{2} \nabla^{2}}{16 \Lambda^{2}}\right) e^{2 \mathcal{F}(V)} e^{2 \boldsymbol{V}}\right)_{i}^{j} \Phi_{j}+\left(\frac{1}{4} M^{i j} \int d^{4} x d^{2} \theta \Phi_{i} \Phi_{j}+\text { c.c. }\right) .
\end{aligned}
$$

The masses of the Pauli-Villars superfields should be proportional to the parameter $\Lambda$ for obtaining a regularized theory with a single dimensionful parameter,

$$
M_{\varphi}=a_{\varphi} \Lambda ; \quad M=a \Lambda,
$$

where the coefficients $a_{\varphi}$ and $a$ are independent of couplings.

\section{Features of quantum corrections in supersymmetric theories regularized by higher derivatives}

Application of the higher covariant derivative regularization to explicit calculations revealed a lot of interesting features of quantum corrections in supersymmetric theories which could not be noted with the dimensional reduction. In particular, the calculation of Ref. [40] demonstrated that the integrals giving the $\beta$-function of $\mathcal{N}=1$ supersymmetric electrodynamics (SQED) are integrals of total derivatives with respect to the loop momenta. In Ref. [41] it was noted that they are also integrals of double total derivatives. The all-order proof of this fact has been done in Refs. [42, 43] and subsequently verified by an explicit three-loop calculation of Ref. [44]. These integrals are similar to the toy integral

$$
\int \frac{d^{4} Q}{(2 \pi)^{4}} \frac{\partial^{2}}{\partial Q_{\mu}^{2}}\left[\frac{f\left(Q^{2}\right)}{Q^{2}}\right]=\frac{1}{8 \pi^{4}} \oint_{S_{\varepsilon}^{3}} d S \frac{1}{Q^{3}}\left(f\left(Q^{2}\right)-Q^{2} f^{\prime}\left(Q^{2}\right)\right)=\frac{1}{4 \pi^{2}} f(0),
$$

where $f\left(Q^{2}\right)$ is a nonsingular function rapidly decreasing at infinity. Due to the factorization into double total derivatives one of loop integrals can be calculated analytically. In the Abelian case this allows to relate the $\beta$-function in a certain order to the anomalous dimension of the matter superfields in the previous order by the so-called exact NSVZ $\beta$-function [45, 46]

$$
\left.\beta\left(\alpha_{0}\right) \equiv \frac{d \alpha_{0}}{d \ln \Lambda}\right|_{\alpha=\text { const }}=\frac{\alpha_{0}^{2} N_{f}}{\pi}\left(1-\gamma\left(\alpha_{0}\right)\right) \equiv \frac{\alpha_{0}^{2} N_{f}}{\pi}\left(1+\left.\frac{d \ln Z}{d \ln \Lambda}\right|_{\alpha=\text { const }}\right),
$$

where $N_{f}$ is a number of flavors [42, 43]. It is important that the renormalization group functions (RGFs) in this equation are defined in terms of the bare coupling constant. Such RGFs are scheme independent for a fixed regularization, so that in the case of using the higher derivative regularization Eq. (15) takes place in all orders for an arbitrary renormalization prescription. In the case of using the dimensional reduction this is not so [47, 48, and Eq. (15) for RGFs 
defined in terms of the bare coupling constant does not take place starting from the three-loop approximation.

For the standard definition of RGFs in terms of the renormalized coupling constant,

$$
\left.\widetilde{\beta}(\alpha) \equiv \frac{d \alpha}{d \ln \mu}\right|_{\alpha_{0}=\text { const }} \quad ;\left.\quad \widetilde{\gamma}(\alpha) \equiv \frac{d \ln Z}{d \ln \mu}\right|_{\alpha_{0}=\text { const }},
$$

the Abelian NSVZ equation is valid only in a certain class of the subtraction schemes described in [49]. It is well known that the $\overline{\mathrm{DR}}$-scheme (i.e. dimensional reduction supplemented by the modified minimal subtractions [50]) does not belong to this class in both Abelian and nonAbelian cases [51, 52, 53, 54, 55].

However, the NSVZ scheme can easily be constructed in all orders in the case of using the higher derivative regularization [56, 57, 58]. It is obtained with the so-called HD+MSL prescription [59, 60, when the theory is regularized by Higher covariant Derivatives and Minimal Subtractions of Logarithms are used for the renormalization. In this case one should include into the renormalization constants only powers of $\ln \Lambda / \mu$. Another example of an all-order prescription which gives the NSVZ scheme in the Abelian case is the on-shell scheme 61. The MOM scheme is not NSVZ [58].

Using the method proposed in Ref. 42 some NSVZ-like relations were also proved in all orders. In particular, the factorization of integrands into double total derivatives turns out to produce the NSVZ-like equation [62, 63, 64] describing running of the photino mass in softly broken SQED [65] and the NSVZ-like relation for the Adler $D$-function in $\mathcal{N}=1$ SQCD [66, 67]. In both cases with the higher covariant derivative regularization RGFs defined in terms of the bare couplings satisfy these equations independently of a renormalization prescripton, while the NSVZ scheme for RGFs defined in terms of the renormalized couplings is given by the HD+MSL prescription, see Refs. 68, and 69. In the case of using dimensional reduction the former RGFs do not satisfy the NSVZ equation, while for the latter RGFs the NSVZ scheme should be specially tuned in each order of the perturbation theory, see Refs. [63] and [70].

However, the generalization of the results obtained in Ref. 42 to the non-Abelian case turned out to be a much more complicated problem. Although numerous explicit calculations made with the higher covariant derivative regularization [71, 72, 73, 74, 75, 76, 77] reveal the structure of loop integrals similar to the Abelian case, the factorization into the double total derivatives has been proved in all orders only recently [78]. (Note that the main ingredient of this proof is again the regularization by higher covariant derivatives.) Moreover, the non-Abelian NSVZ equation [79, 80, 81, 82,

$$
\beta(\alpha, \lambda)=-\frac{\alpha^{2}\left(3 C_{2}-T(R)+C(R)_{i}{ }^{j}\left(\gamma_{\phi}\right)_{j}{ }^{i}(\alpha, \lambda) / r\right)}{2 \pi\left(1-C_{2} \alpha / 2 \pi\right)},
$$

where $r \equiv \operatorname{dim} G, C(R)_{i}{ }^{j} \equiv\left(T^{A} T^{A}\right)_{i}{ }^{j}$, and $C_{2} \equiv T(A d j)$, relates the $\beta$-function to the anomalous dimension of matter superfields in all previous orders, while calculating integrals of double total derivatives we reduce the number of loop integrals only by 1 . The solution of this problem has been found using the new non-renormalization theorem for the triple gauge-ghost vertices proved in Ref. [83] using the Slavnov-Taylor identities [84, 85] and the Feynman rules in $\mathcal{N}=1$ superspace [30, 86, 87, 2 This non-renormalization theorem states that the vertices with two external ghost legs and one external leg of the quantum gauge superfield are finite in all orders of the perturbation theory. This result can be written in the form

\footnotetext{
${ }^{1}$ In Eqs. (17) and (20) we do not specify the definitions of RGFs.

${ }^{2}$ Earlier similar statements were known only in the Landau gauge $\xi \rightarrow 0$ for the (non-supersymmetric) YangMills theory 88] and for $\mathcal{N}=1$ SYM in the Wess-Zumiino gauge [89, while the superfield results of Ref. 83] are valid for the general $\xi$-gauge.
} 


$$
\frac{d}{d \ln \Lambda}\left(Z_{\alpha}^{-1 / 2} Z_{c} Z_{V}\right)=0
$$

where the renormalization constants are defined by the equations

$$
\frac{1}{\alpha_{0}}=\frac{Z_{\alpha}}{\alpha} ; \quad V=Z_{V} Z_{\alpha}^{-1 / 2} V_{R} ; \quad \bar{c} c=Z_{c} Z_{\alpha}^{-1} \bar{c}_{R} c_{R} ; \quad \phi_{i}=\left(\sqrt{Z_{\phi}}\right)_{i}{ }^{j}\left(\phi_{R}\right)_{j}
$$

Therefore, there is a subtraction scheme in which $Z_{\alpha}^{-1 / 2} Z_{c} Z_{V}=1$. This allows rewriting the exact NSVZ $\beta$-function in a new form, which expresses it in terms of the anomalous dimensions of the quantum superfields [83],

$$
\frac{\beta(\alpha, \lambda)}{\alpha^{2}}=-\frac{1}{2 \pi}\left(3 C_{2}-T(R)-2 C_{2} \gamma_{c}(\alpha, \lambda)-2 C_{2} \gamma_{V}(\alpha, \lambda)+C(R)_{i}{ }^{j}\left(\gamma_{\phi}\right)_{j}{ }^{i}(\alpha, \lambda) / r\right)
$$

It is important that this equation relates the $\beta$-function in a certain order only to the anomalous dimensions in the previous order and has the same qualitative interpretation as in the Abelian case. Namely, if we consider a supergraph without external legs, then attaching two external $\boldsymbol{V}$ lines in all possible ways we obtain a (rather large) set of two-point superdiagrams contributing to the function $\beta\left(\alpha_{0}, \lambda_{0}\right) / \alpha_{0}^{2}$. From the other side, by cutting internal lines in the original graph we produce a set of superdiagrams contributing to the anomalous dimensions of the quantum gauge superfield $\gamma_{V}\left(\alpha_{0}, \lambda_{0}\right)$, of the Faddeev-Popov ghost $\gamma_{c}\left(\alpha_{0}, \lambda_{0}\right)$, and of the matter superfields $\left(\gamma_{\phi}\right)_{i}{ }^{j}\left(\alpha_{0}, \lambda_{0}\right)$. Then, all these contributions to various RGFs are related by Eq. (20).

Due to the factorization into double total derivatives Eq. (20) presumably takes place for RGFs defined in terms of the bare couplings in all loops in the case of using the higher covariant derivative regularization. Then according to [83], the NSVZ scheme in the non-Abelian case is given by the HD+MSL prescription. Although the general all-loop proof of these facts is in preparation, the explicit calculations of Refs. [76, 77] confirm them. In these papers the three-loop contributions to the $\beta$-function containing the Yukawa couplings have been compared with the corresponding two-loop contributions to the anomalous dimensions of the quantum superfields. The NSVZ equations (17) and (20) have been checked for RGFs defined in terms of the bare couplings and for RGFs defined in terms of the renormalized couplings in the HD+MSL scheme. It is important that in this approximation the scheme dependence becomes essential 3 Certainly, the corresponding calculations are very complicated from the technical point of view. That is why the complete three-loop calculation with the higher covariant derivative has not yet been done. Actually, only the one-loop calculation with the regularization described above has been made in Ref. [75]. However, according to Ref. [78] it is possible to construct a special method for calculating the $\beta$-function with the higher covariant derivative regularization which simplifies the calculations in a great extent and produces the result in the form of integrals of double total derivatives. Below we formulate the corresponding algorithm and apply it for calculating the two-loop $\beta$-function.

\section{An algorithm for simple calculating the $\beta$-function with the higher covariant derivative regularization}

The proof of the factorization of the loop integrals giving the $\beta$-function into integrals of double total derivatives suggests a simple method for constructing these integrals [78, 90]. It

\footnotetext{
${ }^{3}$ For RGFs defined in terms of the bare couplings the scheme dependence is reduced to the regularization dependence.
} 
is based on the observation that integrals of double total derivatives appear even for a sum of superdiagrams which are formally obtained from a single vacuum supergraph by attaching two external lines of the background gauge superfield in all possible ways. So, let us start with a certain $L$-loop supergraph without external lines and describe an algorithm for obtaining the corresponding contribution to the function $\beta\left(\alpha_{0}, \lambda_{0}\right) / \alpha_{0}^{2}$ :

1. With the help of supersymmetric Feynman rules we should formally write the contribution to the effective action coming from the supergraph under consideration.

2. After this, it is necessary to insert the expression $\theta^{4}\left(v^{B}\right)^{2}$ to an arbitrary point containing the integration over $d^{4} \theta$, where $v^{B}$ are the slowly changing functions which tend to 0 only at a very large scale $R$. If there are no such points, then one should convert one of the integrations over $d^{2} \theta$ into $d^{4} \theta$, which is always possible.

3. Then one should calculate the obtained expression omitting terms suppressed by powers of $1 /(\Lambda R)$ and extract the factor

$$
\mathcal{V}_{4} \equiv \int d^{4} x\left(v^{B}\right)^{2} \rightarrow \infty
$$

4. Next, we mark $L$ propagators corresponding to the Euclidean momenta $Q_{i}^{\mu}$ which are considered as independent. Let $a_{i}$ be the indices corresponding to their beginnings. Evidently, the product of these propagators is proportional to $\prod_{i=1}^{L} \delta_{a_{i}}^{b_{i}}$.

5. The loop integral corresponding to the considered vacuum supergraph is modified by the formal replacement

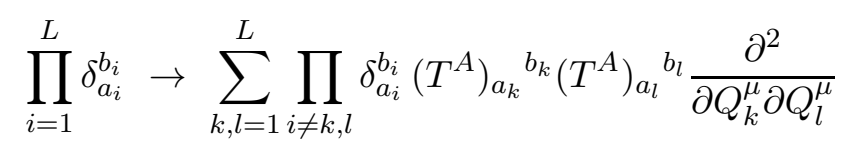

in the integrand.

6. Finally the result is multiplied by

$$
-\frac{2 \pi}{r \mathcal{V}_{4}} \frac{d}{d \ln \Lambda}
$$

Note that the differentiation with respect to $\ln \Lambda$ should be made before the integration over loop momenta to avoid appearing of expressions which diverge in the infrared region.

As a result of the procedure described above we obtain a part of the expression

$$
\frac{1}{\alpha_{0}^{2}}\left(\beta\left(\alpha_{0}, \lambda_{0}\right)-\beta_{1 \text {-loop }}\left(\alpha_{0}\right)\right)
$$

coming from all two-point superdiagrams corresponding to the original vacuum supergraph.

This algorithm simplifies the calculations to a great extent, because instead of calculating a large number of two-point superdiagrams one has to calculate only a single supergraph without external legs.

\section{Two-loop $\beta$-function with the higher derivative regularization}

As an illustration of the method described above, we calculate the two-loop $\beta$-function of the theory (11) in the general $\xi$-gauge (6). It is worth to note that earlier the two-loop $\beta$-function has been obtained only in the Feynman gauge $\xi=1$ in the case of using the simplified version of the higher derivative regularization breaking the BRST invariance. To restore the Slavnov-Taylor identities [84, 85] in this case one had to use a special renormalization procedure proposed in [91, 92] and subsequently generalized to the supersymmetric case in [93, 94]. The result found 
in Ref. 71] using the standard technique of calculations was originally written as a sum of integrals of total derivatives. Subsequently, it was noted that it can be also written in the form of integrals of double total derivatives [74]. Comparing the result with Eq. (20), it is possible to see [59] that Eq. (20) really relates the two-loop $\beta$-function to the one-loop anomalous dimensions of the quantum superfields at the level of loop integrals.

However, the two-loop $\beta$-function has never been calculated with the (BRST invariant) version of the higher covariant derivative regularization described in this paper. Moreover, the gauge dependence of NSVZ equation has not also been investigated. In the case of using the standard methods, the corresponding calculation appears to be rather complicated. However, the technique described above allows making it much easier.

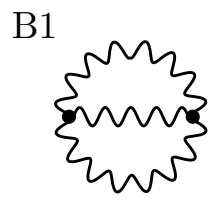

B2

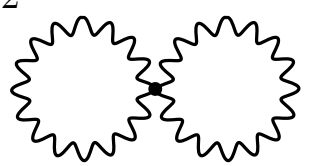

B3

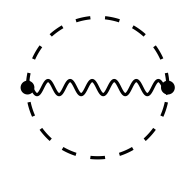

B4

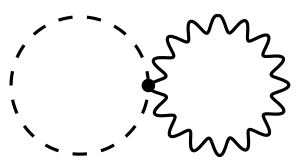

B5

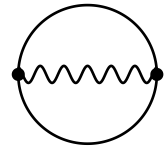

B6

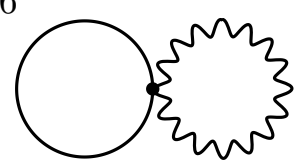

B7

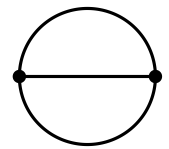

Figure 1: Supergraphs generating the two-loop contributions to the $\beta$-function. The corresponding two-point superdiagrams are obtained from them by attaching two external $\boldsymbol{V}$-legs in all possible ways.

The two-loop contribution to the $\beta$-function is generated by the supergraphs presented in Fig. 1. To obtain (a very large number of) usual two-point superdiagrams, one should attach to them two external legs of the background gauge superfield in all possible ways. However, as we discussed earlier, in the case of using the higher covariant derivative regularization their contributions can be constructed by calculating only specially modified vacuum supergraphs presented in Fig. 1) The result of this calculation obtained according to the algorithm described above can be written as

$$
\begin{aligned}
& \frac{\beta\left(\alpha_{0}, \lambda_{0}\right)}{\alpha_{0}^{2}}-\frac{\beta_{1 \text {-loop }}\left(\alpha_{0}\right)}{\alpha_{0}^{2}}=\Delta_{\text {gauge }+ \text { ghost }}\left(\frac{\beta}{\alpha_{0}^{2}}\right)+\Delta_{\varphi}\left(\frac{\beta}{\alpha_{0}^{2}}\right)+\Delta_{\text {matter }}\left(\frac{\beta}{\alpha_{0}^{2}}\right)+\Delta_{\text {Yukawa }}\left(\frac{\beta}{\alpha_{0}^{2}}\right) \\
& +O\left(\alpha_{0}^{2}, \alpha_{0} \lambda_{0}^{2}, \lambda_{0}^{4}\right),
\end{aligned}
$$

where the expressions for all $\Delta_{I}\left(\beta / \alpha_{0}^{2}\right)$ are presented in Appendix $\mathrm{A}$ and

$$
\beta_{1 \text {-loop }}\left(\alpha_{0}\right)=-\frac{\alpha_{0}^{2}}{2 \pi}\left(3 C_{2}-T(R)\right)
$$

is the one-loop contribution to the $\beta$-function. With the regularization considered in this paper it has been calculated in Ref. [75]. The contributions $\Delta_{\text {gauge+ghost }}\left(\beta / \alpha_{0}^{2}\right), \Delta_{\text {matter }}\left(\beta / \alpha_{0}^{2}\right)$, and $\Delta_{\text {Yukawa }}\left(\beta / \alpha_{0}^{2}\right)$ are the sums of the superdiagrams generated by the supergraphs B1 - B4, B5

\footnotetext{
${ }^{4}$ Similar calculations with different non-invariant versions of the higher derivative regularization can be found in Refs. [72, 73].
} 
and B6 (including the supergraphs containing a loop of the Pauli-Villars superfields $\Phi_{i}$ ), and B7, respectively. The contribution $\Delta_{\varphi}\left(\beta / \alpha_{0}^{2}\right)$ comes from the supergraphs B5 and B6 in which the solid line corresponds to a loop of the Pauli-Villars superfields $\varphi_{a}$.

It is necessary to note that all gauge dependent terms containing the parameter $\xi_{0}$ and the regulator $K(x)$ (see Eq. (6) ) cancel each other, so that the resulting expression turns out to be gauge independent. However, the expressions for separate supergraphs contain gauge dependent terms. (For example, the results for the supergraphs B3 and B4 explicitly written in Ref. 90] for the particular case $K(x)=R(x)$ do contain them.) Also it should be noted that the expressions for separate supergraphs contain some terms which are not well defined. However, in their sum all bad terms disappear and the resulting expression is well defined.

With the help of equations similar to (14) it is possible to calculate one of the loop integrals in the expressions (38) - (41). Omitting inessential higher order terms we obtain

$$
\begin{aligned}
& \Delta_{\text {gauge+ghost }}\left(\frac{\beta}{\alpha_{0}^{2}}\right)=\alpha_{0} C_{2}^{2} \int \frac{d^{4} Q}{(2 \pi)^{4}} \frac{d}{d \ln \Lambda} \frac{\partial^{2}}{\partial Q_{\mu}^{2}}\left[\frac{1}{Q^{2}} \ln R_{Q}\right] \text {; } \\
& \Delta_{\varphi}\left(\frac{\beta}{\alpha_{0}^{2}}\right)=-\alpha_{0} C_{2}^{2} \int \frac{d^{4} Q}{(2 \pi)^{4}} \frac{d}{d \ln \Lambda} \frac{\partial^{2}}{\partial Q_{\mu}^{2}}\left[\frac{1}{Q^{2}} \ln \left(1+\frac{M_{\varphi}^{2}}{Q^{2}}\right)\right. \\
& \left.+\frac{1}{2 Q^{2}} \ln \left(1+\frac{M_{\varphi}^{2}}{Q^{2} R_{Q}^{2}}\right)+\frac{1}{Q^{2}} \ln R_{Q}\right] ; \\
& \Delta_{\text {matter }}\left(\frac{\beta}{\alpha_{0}^{2}}\right)=\frac{4 \alpha_{0}}{r} \operatorname{tr}\left(C(R)^{2}\right) \int \frac{d^{4} Q}{(2 \pi)^{4}} \frac{d}{d \ln \Lambda} \frac{1}{Q^{4} R_{Q}} \\
& +\frac{\alpha_{0}}{2 r} C_{2} \operatorname{tr} C(R) \int \frac{d^{4} Q}{(2 \pi)^{4}} \frac{d}{d \ln \Lambda} \frac{\partial^{2}}{\partial Q_{\mu}^{2}}\left[\frac{1}{Q^{2}} \ln \left(1+\frac{M^{2}}{Q^{2} F_{Q}^{2}}\right)\right] \text {; } \\
& \Delta_{\text {Yukawa }}\left(\frac{\beta}{\alpha_{0}^{2}}\right)=-\frac{1}{\pi r} \lambda_{0 j m n}^{*} \lambda_{0}^{i m n} C(R)_{i}{ }^{j} \int \frac{d^{4} Q}{(2 \pi)^{4}} \frac{d}{d \ln \Lambda} \frac{1}{Q^{4} F_{Q}^{2}} \text {. }
\end{aligned}
$$

Summing up these contributions and substituting the expression (26) for the one-loop $\beta$-function, we find the complete expression for the two-loop $\beta$-function in the form of the loop integrals

$$
\begin{aligned}
& \frac{\beta\left(\alpha_{0}, \lambda_{0}\right)}{\alpha_{0}^{2}}=-\frac{1}{2 \pi}\left(3 C_{2}-T(R)\right)-\alpha_{0} C_{2}^{2} \int \frac{d^{4} Q}{(2 \pi)^{4}} \frac{d}{d \ln \Lambda} \frac{\partial^{2}}{\partial Q_{\mu}^{2}}\left[\frac{1}{2 Q^{2}} \ln \left(1+\frac{M_{\varphi}^{2}}{Q^{2} R_{Q}^{2}}\right)\right. \\
& \left.+\frac{1}{Q^{2}} \ln \left(1+\frac{M_{\varphi}^{2}}{Q^{2}}\right)\right]+\frac{\alpha_{0}}{2 r} C_{2} \operatorname{tr} C(R) \int \frac{d^{4} Q}{(2 \pi)^{4}} \frac{d}{d \ln \Lambda} \frac{\partial^{2}}{\partial Q_{\mu}^{2}}\left[\frac{1}{Q^{2}} \ln \left(1+\frac{M^{2}}{Q^{2} F_{Q}^{2}}\right)\right] \\
& +\frac{4 \alpha_{0}}{r} \operatorname{tr}\left(C(R)^{2}\right) \int \frac{d^{4} Q}{(2 \pi)^{4}} \frac{d}{d \ln \Lambda} \frac{1}{Q^{4} R_{Q}}-\frac{1}{\pi r} \lambda_{0 j m n}^{*} \lambda_{0}^{i m n} C(R)_{i}{ }^{j} \int \frac{d^{4} Q}{(2 \pi)^{4}} \frac{d}{d \ln \Lambda} \frac{1}{Q^{4} F_{Q}^{2}} \\
& +O\left(\alpha_{0}^{2}, \alpha_{0} \lambda_{0}^{2}, \lambda_{0}^{4}\right) .
\end{aligned}
$$

Before calculating the remaining integrals, it is expedient to compare this expression with the one-loop anomalous dimensions of the quantum superfields (defined in terms of the bare couplings) obtained in Ref. [75],

$$
\begin{aligned}
\gamma_{V} & =-\pi \alpha_{0} \int \frac{d^{4} Q}{(2 \pi)^{4}} \frac{d}{d \ln \Lambda} \frac{\partial^{2}}{\partial Q_{\mu}^{2}}\left[\frac{C_{2}}{2 Q^{2}} \ln \left(1+\frac{M_{\varphi}^{2}}{Q^{2} R_{Q}^{2}}\right)+\frac{C_{2}}{Q^{2}} \ln \left(1+\frac{M_{\varphi}^{2}}{Q^{2}}\right)-\frac{1}{2 r} \operatorname{tr} C(R) \frac{1}{Q^{2}}\right. \\
& \left.\times \ln \left(1+\frac{M^{2}}{Q^{2} F_{Q}^{2}}\right)\right]+4 \pi \alpha_{0} C_{2} \int \frac{d^{4} Q}{(2 \pi)^{4}} \frac{d}{d \ln \Lambda}\left(\frac{1}{3 Q^{4} R_{Q}}-\frac{\xi_{0}}{3 Q^{4} K_{Q}}\right)+O\left(\alpha_{0}^{2}, \alpha_{0} \lambda_{0}^{2}\right) ;
\end{aligned}
$$




$$
\begin{aligned}
& \gamma_{c}=4 \pi \alpha_{0} C_{2} \int \frac{d^{4} Q}{(2 \pi)^{4}} \frac{d}{d \ln \Lambda}\left(-\frac{1}{3 Q^{4} R_{Q}}+\frac{\xi_{0}}{3 Q^{4} K_{Q}}\right)+O\left(\alpha_{0}^{2}, \alpha_{0} \lambda_{0}^{2}\right) \\
& \left(\gamma_{\phi}\right)_{i}{ }^{j}=\int \frac{d^{4} Q}{(2 \pi)^{4}} \frac{d}{d \ln \Lambda}\left(-C(R)_{i}{ }^{j} \frac{8 \pi \alpha_{0}}{Q^{4} R_{Q}}+\lambda_{0 i m n}^{*} \lambda_{0}^{j m n} \frac{2}{Q^{4} F_{Q}^{2}}\right)+O\left(\alpha_{0}^{2}, \alpha_{0} \lambda_{0}^{2}, \lambda_{0}^{4}\right) .
\end{aligned}
$$

Then we see that even at the level of the loop integrals the NSVZ equation in the form (20) is satisfied by these RGFs in the considered approximation,

$$
\begin{aligned}
& \frac{\beta\left(\alpha_{0}, \lambda_{0}\right)}{\alpha_{0}^{2}}=-\frac{1}{2 \pi}\left(3 C_{2}-T(R)-2 C_{2} \gamma_{c}\left(\alpha_{0}, \lambda_{0}\right)-2 C_{2} \gamma_{V}\left(\alpha_{0}, \lambda_{0}\right)+C(R)_{i}{ }^{j}\left(\gamma_{\phi}\right)_{j}{ }^{i}\left(\alpha_{0}, \lambda_{0}\right) / r\right) \\
& +O\left(\alpha_{0}^{2}, \alpha_{0} \lambda_{0}^{2}, \lambda_{0}^{4}\right)=-\frac{1}{2 \pi}\left(3 C_{2}-T(R)\right)+\frac{\alpha_{0}}{(2 \pi)^{2}}\left(-3 C_{2}^{2}+\frac{1}{r} C_{2} \operatorname{tr} C(R)+\frac{2}{r} \operatorname{tr}\left(C(R)^{2}\right)\right) \\
& -\frac{1}{8 \pi^{3} r} C(R)_{i}{ }^{j} \lambda_{0 j m n}^{*} \lambda_{0}^{i m n}+O\left(\alpha_{0}^{2}, \alpha_{0} \lambda_{0}^{2}, \lambda_{0}^{4}\right) .
\end{aligned}
$$

It is easy to verify that (up to notations) this result agrees with the first two-loop calculation made in Ref. [95] with dimensional regularization. Certainly, this occurs due to the scheme independence of the two-loop $\beta$-function.

Note that, according to [75], at the level of loop integrals

$$
\gamma_{V}\left(\alpha_{0}, \lambda_{0}\right)+\gamma_{c}\left(\alpha_{0}, \lambda_{0}\right)=\frac{\beta\left(\alpha_{0}, \lambda_{0}\right)}{2 \alpha_{0}}+O\left(\alpha_{0}^{2}, \alpha_{0} \lambda_{0}^{2}\right)
$$

This implies that the original NSVZ equation (17) is also valid for RGFs defined in terms of the bare couplings in the considered approximation,

$$
\frac{\beta\left(\alpha_{0}, \lambda_{0}\right)}{\alpha_{0}^{2}}=-\frac{3 C_{2}-T(R)+C(R)_{i}{ }^{j}\left(\gamma_{\phi}\right)_{j}{ }^{i}\left(\alpha_{0}, \lambda_{0}\right) / r}{2 \pi\left(1-C_{2} \alpha_{0} / 2 \pi\right)}+O\left(\alpha_{0}^{2}, \alpha_{0} \lambda_{0}^{2}, \lambda_{0}^{4}\right) .
$$

This result is analogous to the one obtained in Ref. [59] in the case of using the simplified BRST non-invariant version of the higher derivative regularization. However, using of the invariant regularization is certainly more preferable. Moreover, Eq. (35) has been derived for the general $\xi$-gauge (6). This allows investigating the gauge dependence of the NSVZ equation. We see that the $\beta$-function is gauge independent, while the anomalous dimensions (32) and (33) explicitly depend on the gauge parameter $\xi_{0}$. However, this gauge dependence disappears in the sum of terms in the right hand side. This implies that the NSVZ equation takes place in an arbitrary $\xi$-gauge.

Taking into account that the two-loop $\beta$-function and the one-loop anomalous dimensions are scheme independent, we also see that in the considered approximation the NSVZ equations (17) and (20) are also satisfied by RGFs defined in terms of the renormalized couplings for an arbitrary renormalization prescription.

\section{Conclusion}

In this paper we argue that the Slavnov higher covariant derivative regularization is a very useful tool for both investigating the structure of quantum corrections in supersymmetric theories and explicit perturbative calculations. Unlike the dimensional reduction, it allows to reveal the origin of the exact NSVZ $\beta$-function, namely, the factorization of integrals giving the $\beta$-function into integrals of double total derivatives with respect to the loop momenta. Due to this fact RGFs defined in terms of the bare couplings satisfy the NSVZ equation in all loops independently 
of a renormalization prescription. For RGFs defined in terms of the renormalized couplings the NSVZ scheme in all orders is given by the HD+MSL prescription, which in particular includes this regularization. Note that the proof of these facts essentially involves the new nonrenormalization theorem for the triple gauge-ghost vertices. This theorem was also derived with the help of the higher covariant derivative regularization using the supergraph calculation rules and the Slavnov-Taylor identities.

Last years a large number of explicit multiloop calculations were made with the higher covariant derivative regularization. These calculations confirm the general statements discussed above. In particular, the statement "HD+MSL=NSVZ" has been checked in such an order of the perturbation theory where the scheme dependence is essential. Note that recently a special method for making such calculations was proposed [78. It allows to simplify the calculations considerably and to obtain the results for contributions to $\beta$-function in the form of integrals of double total derivatives. In this paper we demonstrated the application of this method by calculating the two-loop $\beta$-function for the general renormalizable $\mathcal{N}=1$ supersymmetric gauge theory in the $\xi$-gauge. This calculation confirmed the validity of the new form of the NSVZ equation (20) at the level of loop integrals for an arbitrary $\xi$ and showed the gauge independence of both sides of the NSVZ equation, at least, in the considered approximation.

\section{Acknowledgements}

This work was supported by Foundation for Advancement of Theoretical Physics and Mathematics "BASIS", grant No. 19-1-1-45-1.

\section{Appendix}

\section{A Contributions of various two-loop supergraphs to the $\beta$ - function}

In this appendix we collect the expressions for contributions of the supergraphs B1 - B7 to the function $\beta / \alpha_{0}^{2}$, which were obtained with the help of the method described in Sect. 4. Certainly, by construction, the results are given by integrals of double total derivatives.

$$
\begin{aligned}
& \Delta_{\text {gauge }+ \text { ghost }}\left(\frac{\beta}{\alpha_{0}^{2}}\right)=4 \pi C_{2}^{2} \frac{d}{d \ln \Lambda} \int \frac{d^{4} Q}{(2 \pi)^{4}} \frac{d^{4} K}{(2 \pi)^{4}}\left[\frac{\partial^{2}}{\partial Q_{\mu}^{2}}+\frac{\partial^{2}}{\partial K_{\mu}^{2}}-\frac{\partial^{2}}{\partial Q_{\mu} \partial K^{\mu}}\right] \frac{e_{0}^{2}}{R_{K} R_{Q}} \\
& \times\left\{-\frac{R_{K}}{2 Q^{2} K^{2}(K+Q)^{2}}-\frac{1}{2 Q^{2} K^{2}}\left(\frac{R_{Q}-R_{K}}{Q^{2}-K^{2}}\right)-\frac{1}{R_{K+Q} K^{2}}\left(1-\frac{Q^{2}}{2(K+Q)^{2}}\right)\left(\frac{R_{Q}-R_{K}}{Q^{2}-K^{2}}\right)\right. \\
& \times\left(\frac{R_{K+Q}-R_{Q}}{(K+Q)^{2}-Q^{2}}\right)-\frac{1}{R_{K+Q}(K+Q)^{2}}\left(\frac{R_{Q}-R_{K}}{Q^{2}-K^{2}}\right)^{2}+\frac{2}{K^{2}\left((K+Q)^{2}-Q^{2}\right)^{2}} \\
& \times\left[R_{K+Q}-R_{Q}-R_{Q}^{\prime}\left(\frac{(K+Q)^{2}}{\Lambda^{2}}-\frac{Q^{2}}{\Lambda^{2}}\right)\right]-\frac{Q_{\mu} K^{\mu}}{Q^{2} K^{2}}\left[\frac{R_{K+Q}}{\left((K+Q)^{2}-K^{2}\right)\left((K+Q)^{2}-Q^{2}\right)}\right. \\
& \left.\left.+\frac{R_{Q}}{\left(K^{2}-(K+Q)^{2}\right)\left(K^{2}-Q^{2}\right)}+\frac{R_{Q}}{\left(Q^{2}-(K+Q)^{2}\right)\left(Q^{2}-K^{2}\right)}\right]\right\} ; \\
& \Delta_{\varphi}\left(\frac{\beta}{\alpha_{0}^{2}}\right)=4 \pi C_{2}^{2} \frac{d}{d \ln \Lambda} \int \frac{d^{4} Q}{(2 \pi)^{4}} \frac{d^{4} K}{(2 \pi)^{4}}\left[\frac{\partial^{2}}{\partial Q_{\mu}^{2}}+\frac{\partial^{2}}{\partial K_{\mu}^{2}}-\frac{\partial^{2}}{\partial Q_{\mu} \partial K^{\mu}}\right] \frac{e_{0}^{2}}{K^{2} R_{K}}\left\{\frac{1}{\left(Q^{2}+M_{\varphi}^{2}\right)}\right.
\end{aligned}
$$




$$
\begin{aligned}
& \times \frac{1}{\left((Q+K)^{2}+M_{\varphi}^{2}\right)}-\frac{1}{\left((K+Q)^{2}-Q^{2}\right)}\left[\frac{R_{K+Q}^{2}}{2\left((K+Q)^{2} R_{K+Q}^{2}+M_{\varphi}^{2}\right)}-\frac{R_{Q}^{2}}{2\left(Q^{2} R_{Q}^{2}+M_{\varphi}^{2}\right)}\right. \\
& \left.\left.-\frac{M_{\varphi}^{2} R_{K+Q}^{\prime}}{\Lambda^{2} R_{K+Q}\left((K+Q)^{2} R_{K+Q}^{2}+M_{\varphi}^{2}\right)}+\frac{M_{\varphi}^{2} R_{Q}^{\prime}}{\Lambda^{2} R_{Q}\left(Q^{2} R_{Q}^{2}+M_{\varphi}^{2}\right)}+\frac{R_{K+Q}^{\prime}}{\Lambda^{2} R_{K+Q}}-\frac{R_{Q}^{\prime}}{\Lambda^{2} R_{Q}}\right]\right\} ; \quad(39) \\
& \Delta_{\text {matter }}\left(\frac{\beta}{\alpha_{0}^{2}}\right)=\frac{4 \pi}{r} \frac{d}{d \ln \Lambda} \int \frac{d^{4} Q}{(2 \pi)^{4}} \frac{d^{4} K}{(2 \pi)^{4}}\left[\operatorname{tr}\left(C(R)^{2}\right) \frac{\partial^{2}}{\partial Q_{\mu}^{2}}+C_{2} \operatorname{tr} C(R)\left(\frac{\partial^{2}}{\partial K_{\mu}^{2}}-\frac{\partial^{2}}{\partial Q_{\mu} \partial K^{\mu}}\right)\right] \\
& \times \frac{e_{0}^{2}}{K^{2} R_{K}}\left\{\frac{1}{2 Q^{2}(Q+K)^{2}}+\frac{F_{K+Q}^{2}}{\left((K+Q)^{2}-Q^{2}\right)}\left[\frac{F_{Q}^{2}}{2\left((K+Q)^{2} F_{K+Q}^{2}+M^{2}\right)}-\frac{M^{2} F_{Q}^{\prime}}{2\left(Q^{2} F_{Q}^{2}+M^{2}\right)}\right.\right. \\
& \left.\left.-\frac{M^{2} F_{K+Q}^{\prime}}{\Lambda^{2} F_{K+Q}\left((K+Q)^{2} F_{K+Q}^{2}+M^{2}\right)}+\frac{1}{\Lambda^{2} F_{Q}\left(Q^{2} F_{Q}^{2}+M^{2}\right)}\right]\right\} ; \\
& \Delta_{\text {Yukawa }}\left(\frac{\beta}{\alpha_{0}^{2}}\right)=-\frac{2 \pi}{r} \frac{d}{d \ln \Lambda} \int \frac{d^{4} Q}{(2 \pi)^{4}} \frac{d^{4} K}{(2 \pi)^{4}} \lambda_{0}^{i j k} \lambda_{0 i j l}^{*} C(R)_{k}{ }^{l} \frac{\partial^{2}}{\partial Q_{\mu}^{2}}\left(\frac{1}{Q^{2} F_{Q} K^{2} F_{K}(Q+K)^{2} F_{Q+K}}\right) .
\end{aligned}
$$

\section{References}

[1] M. E. Peskin and D. V. Schroeder, "An Introduction to quantum field theory," CRC Press, Taylor \& Francis group (2019) 866 p.

[2] R. N. Mohapatra, "Unification And Supersymmetry. The Frontiers Of Quark - Lepton Physics : The Frontiers Of Quark-lepton Physics," New York, USA: Springer (2003) 421 p.

[3] G. 't Hooft and M. J. G. Veltman, Nucl. Phys. B 44 (1972) 189.

[4] C. G. Bollini and J. J. Giambiagi, Nuovo Cim. B 12 (1972) 20.

[5] J. F. Ashmore, Lett. Nuovo Cim. 4 (1972) 289.

[6] G. M. Cicuta and E. Montaldi, Lett. Nuovo Cim. 4 (1972) 329.

[7] R. Delbourgo and V. B. Prasad, J. Phys. G 1 (1975) 377.

[8] W. Siegel, Phys. Lett. 84B (1979) 193.

[9] W. Siegel, Phys. Lett. 94B (1980) 37.

[10] L. V. Avdeev, G. A. Chochia and A. A. Vladimirov, Phys. Lett. 105B (1981) 272.

[11] L. V. Avdeev and A. A. Vladimirov, Nucl. Phys. B 219 (1983) 262.

[12] A. A. Slavnov, Nucl. Phys. B 31 (1971) 301.

[13] A. A. Slavnov, Theor.Math.Phys. 13 (1972) 1064 [Teor. Mat. Fiz. 13 (1972) 174].

[14] V. K. Krivoshchekov, Theor. Math. Phys. 36 (1978) 745 [Teor. Mat. Fiz. 36 (1978) 291].

[15] P. C. West, Nucl. Phys. B 268 (1986) 113.

[16] I. L. Buchbinder and K. V. Stepanyantz, Nucl. Phys. B 883 (2014) 20.

[17] A. Galperin, E. Ivanov, S. Kalitzin, V. Ogievetsky and E. Sokatchev, Class. Quant. Grav. 1 (1984) 469-498 [Corrigendum ibid. 2 (1985) 127]. 
[18] A. S. Galperin, E. A. Ivanov, V. I. Ogievetsky and E. S. Sokatchev, "Harmonic superspace", Cambridge, UK: Univ. Pr. (2001) 306 p.

[19] I. L. Buchbinder, N. G. Pletnev and K. V. Stepanyantz, Phys. Lett. B 751 (2015) 434.

[20] M. T. Grisaru and W. Siegel, Nucl. Phys. B 201 (1982) 292 Erratum: [Nucl. Phys. B 206 (1982) 496].

[21] S. Mandelstam, Nucl. Phys. B 213 (1983) 149.

[22] L. Brink, O. Lindgren and B. E. W. Nilsson, Nucl. Phys. B 212 (1983) 401.

[23] P. S. Howe, K. S. Stelle and P. K. Townsend, Nucl. Phys. B 236 (1984) 125.

[24] I. L. Buchbinder, S. M. Kuzenko and B. A. Ovrut, Phys. Lett. B 433 (1998) 335.

[25] L. D. Faddeev and A. A. Slavnov, Front. Phys. 50 (1980) 1 [Front. Phys. (1991) 1].

[26] A. A. Slavnov, Theor. Math. Phys. 33 (1977) 977 [Teor. Mat. Fiz. 33 (1977) 210].

[27] B. S. DeWitt, "Dynamical theory of groups and fields," Gordon and Breach, New York, 1965.

[28] L. F. Abbott, Nucl. Phys. B 185 (1981) 189.

[29] L. F. Abbott, Acta Phys. Polon. B 13 (1982) 33.

[30] S. J. Gates, M. T. Grisaru, M. Rocek and W. Siegel, Front. Phys. 58 (1983) 1.

[31] O. Piguet and K. Sibold, Nucl. Phys. B 197 (1982) 257.

[32] O. Piguet and K. Sibold, Nucl. Phys. B 197 (1982) 272.

[33] I. V. Tyutin, Yad. Fiz. 37 (1983) 761.

[34] J. W. Juer and D. Storey, Phys. Lett. 119B (1982) 125.

[35] J. W. Juer and D. Storey, Nucl. Phys. B 216 (1983) 185.

[36] A. E. Kazantsev, M. D. Kuzmichev, N. P. Meshcheriakov, S. V. Novgorodtsev, I. E. Shirokov, M. B. Skoptsov and K. V. Stepanyantz, JHEP 1806 (2018) 020.

[37] C. Becchi, A. Rouet and R. Stora, Commun. Math. Phys. 42 (1975) 127.

[38] I. V. Tyutin, "Gauge Invariance in Field Theory and Statistical Physics in Operator Formalism," arXiv:0812.0580 [hep-th].

[39] A. E. Kazantsev, M. B. Skoptsov and K. V. Stepanyantz, Mod. Phys. Lett. A 32 (2017) no.36, 1750194.

[40] A. A. Soloshenko and K. V. Stepanyantz, Theor. Math. Phys. 140 (2004) 1264 [Teor. Mat. Fiz. 140 (2004) 437].

[41] A. V. Smilga and A. Vainshtein, Nucl. Phys. B 704 (2005) 445.

[42] K. V. Stepanyantz, Nucl. Phys. B 852 (2011) 71.

[43] K. V. Stepanyantz, JHEP 1408 (2014) 096. 
[44] A. E. Kazantsev and K. V. Stepanyantz, J. Exp. Theor. Phys. 120 (2015) no.4, 618 [Zh. Eksp. Teor. Fiz. 147 (2015) no.4, 714].

[45] A. I. Vainshtein, V. I. Zakharov and M. A. Shifman, JETP Lett. 42 (1985) 224 [Pisma Zh. Eksp. Teor. Fiz. 42 (1985) 182].

[46] M. A. Shifman, A. I. Vainshtein and V. I. Zakharov, Phys. Lett. 166B (1986) 334.

[47] S. S. Aleshin, A. L. Kataev and K. V. Stepanyantz, JETP Lett. 103 (2016) no.2, 77.

[48] S. S. Aleshin, I. O. Goriachuk, A. L. Kataev and K. V. Stepanyantz, Phys. Lett. B 764 (2017) 222.

[49] I. O. Goriachuk, A. L. Kataev and K. V. Stepanyantz, Phys. Lett. B 785 (2018) 561.

[50] W. A. Bardeen, A. J. Buras, D. W. Duke and T. Muta, Phys. Rev. D 18 (1978) 3998.

[51] I. Jack, D. R. T. Jones and C. G. North, Phys. Lett. B 386 (1996) 138.

[52] I. Jack, D. R. T. Jones and C. G. North, Nucl. Phys. B 486 (1997) 479.

[53] I. Jack, D. R. T. Jones and A. Pickering, Phys. Lett. B 435 (1998) 61.

[54] R. V. Harlander, D. R. T. Jones, P. Kant, L. Mihaila and M. Steinhauser, JHEP 0612 (2006) 024.

[55] L. Mihaila, Adv. High Energy Phys. 2013 (2013) 607807.

[56] A. L. Kataev and K. V. Stepanyantz, Nucl. Phys. B 875 (2013) 459.

[57] A. L. Kataev and K. V. Stepanyantz, Phys. Lett. B 730 (2014) 184.

[58] A. L. Kataev and K. V. Stepanyantz, Theor. Math. Phys. 181 (2014) 1531.

[59] V. Y. Shakhmanov and K. V. Stepanyantz, Phys. Lett. B 776 (2018) 417.

[60] K. V. Stepanyantz, Bled Workshops Phys. 18 (2017) no.2, 197.

[61] A. L. Kataev, A. E. Kazantsev and K. V. Stepanyantz, Eur. Phys. J. C 79 (2019) no.6, 477.

[62] J. Hisano and M. A. Shifman, Phys. Rev. D 56 (1997) 5475.

[63] I. Jack and D. R. T. Jones, Phys. Lett. B 415 (1997) 383.

[64] L. V. Avdeev, D. I. Kazakov and I. N. Kondrashuk, Nucl. Phys. B 510 (1998) 289.

[65] I. V. Nartsev and K. V. Stepanyantz, JHEP 1704 (2017) 047.

[66] M. Shifman and K. Stepanyantz, Phys. Rev. Lett. 114 (2015) no.5, 051601.

[67] M. Shifman and K. V. Stepanyantz, Phys. Rev. D 91 (2015) 105008.

[68] I. V. Nartsev and K. V. Stepanyantz, JETP Lett. 105 (2017) no.2, 69.

[69] A. L. Kataev, A. E. Kazantsev and K. V. Stepanyantz, Nucl. Phys. B 926 (2018) 295.

[70] S. S. Aleshin, A. L. Kataev and K. V. Stepanyantz, JHEP 1903 (2019) 196.

[71] A. B. Pimenov, E. S. Shevtsova and K. V. Stepanyantz, Phys. Lett. B 686 (2010) 293. 
[72] K. V. Stepanyantz, Proc. Steklov Inst. Math. 272 (2011) no.1, 256.

[73] K. V. Stepanyantz, Phys. Part. Nucl. Lett. 8 (2011) 321.

[74] K. V. Stepanyantz, "Factorization of integrals defining the two-loop $\beta$-function for the general renormalizable $\mathrm{N}=1 \mathrm{SYM}$ theory, regularized by the higher covariant derivatives, into integrals of double total derivatives," arXiv:1108.1491 [hep-th].

[75] S. S. Aleshin, A. E. Kazantsev, M. B. Skoptsov and K. V. Stepanyantz, JHEP 1605 (2016) 014.

[76] V. Y. Shakhmanov and K. V. Stepanyantz, Nucl. Phys. B 920 (2017) 345.

[77] A. E. Kazantsev, V. Y. Shakhmanov and K. V. Stepanyantz, JHEP 1804 (2018) 130.

[78] K. V. Stepanyantz, JHEP 1910 (2019) 011.

[79] V. A. Novikov, M. A. Shifman, A. I. Vainshtein and V. I. Zakharov, Nucl. Phys. B 229 (1983) 381.

[80] D. R. T. Jones, Phys. Lett. 123B (1983) 45.

[81] V. A. Novikov, M. A. Shifman, A. I. Vainshtein and V. I. Zakharov, Phys. Lett. 166B (1986) 329 [Sov. J. Nucl. Phys. 43 (1986) 294] [Yad. Fiz. 43 (1986) 459].

[82] M. A. Shifman and A. I. Vainshtein, Nucl. Phys. B 277 (1986) 456 [Sov. Phys. JETP 64 (1986) 428] [Zh. Eksp. Teor. Fiz. 91 (1986) 723].

[83] K. V. Stepanyantz, Nucl. Phys. B 909 (2016) 316.

[84] J. C. Taylor, Nucl. Phys. B 33 (1971) 436.

[85] A. A. Slavnov, Theor. Math. Phys. 10 (1972) 99 [Teor. Mat. Fiz. 10 (1972) 153].

[86] P. C. West, "Introduction to supersymmetry and supergravity," Singapore, Singapore: World Scientific (1990) 425 p.

[87] I. L. Buchbinder and S. M. Kuzenko, "Ideas and methods of supersymmetry and supergravity: Or a walk through superspace," Bristol, UK: IOP (1998) 656 p.

[88] D. Dudal, H. Verschelde and S. P. Sorella, Phys. Lett. B 555 (2003) 126.

[89] M. A. L. Capri, D. R. Granado, M. S. Guimaraes, I. F. Justo, L. Mihaila, S. P. Sorella and D. Vercauteren, Eur. Phys. J. C 74 (2014) no.4, 2844.

[90] M. D. Kuzmichev, N. P. Meshcheriakov, S. V. Novgorodtsev, I. E. Shirokov and K. V. Stepanyantz, Eur. Phys. J. C 79 (2019) no.9, 809.

[91] A. A. Slavnov, Phys. Lett. B 518 (2001) 195.

[92] A. A. Slavnov, Theor. Math. Phys. 130 (2002) 1 [Teor. Mat. Fiz. 130 (2002) 3].

[93] A. A. Slavnov and K. V. Stepanyantz, Theor. Math. Phys. 135 (2003) 673 [Teor. Mat. Fiz. 135 (2003) 265].

[94] A. A. Slavnov and K. V. Stepanyantz, Theor. Math. Phys. 139 (2004) 599 [Teor. Mat. Fiz. 139 (2004) 179].

[95] D. R. T. Jones, Nucl. Phys. B 87 (1975) 127. 